\title{
Experimental Verification of the Aerodynamics of Stream Thrust Probes*
}

\author{
Robert S. Hiers III" and James R. Sirbaugh ${ }^{\star}$ \\ Aerospace Testing Alliance (ATA), Arnold Engineering Development Center, Arnold Air Force Base, TN 37389 \\ and \\ Capt Barrett T. McCann, ${ }^{\S}$ Russell M. Cummings, ${ }^{* *}$ Cadet Laura S. Luft, ${ }^{\dagger \dagger}$ and Cadet Dimitrios P. Grillos ${ }^{\sharp}$ \\ United States Air Force Academy, USAF Academy, Colorado 80840
}

\begin{abstract}
Determining the local stream thrust (a vector quantity) from a measured pitot pressure (a scalar quantity) requires either knowledge of the flow direction or a probe shape that compensates for flow direction. This compensation ideally would make the measured pressure directly proportional to the component of momentum along the probe axis. The flow angle sensitivity required to resolve this component of momentum was previously determined theoretically. A proposed probe nose shape was analyzed using computational fluid dynamics (CFD) and was found to produce a flow angle sensitivity close to the required sensitivity. In the current work, the proposed nose shape was tested in a wind tunnel at Mach numbers 1.67, 2.45, and 3.48 at angles of attack from 0 to $15 \mathrm{deg}$. The test results indicate that the flow angle sensitivity of the proposed nose shape agrees with the required sensitivity to within 1 percent up to a flow angle of $15 \mathrm{deg}$.
\end{abstract}

\section{Introduction}

In a previous paper, ${ }^{1}$ a derivation was given to determine the local stream thrust from a measured pitot pressure. Ideal, perfect gas behavior was assumed in order to illustrate the technique, which requires a probe shape that automatically compensates for flow direction. ${ }^{2}$ A computational fluid dynamics (CFD) study compared the flow angle compensation characteristics of two proposed probe geometries. ${ }^{2}$ In the present paper, the flow angle sensitivity of one of the probe shapes is investigated experimentally. The probe nose shape is designed such that the measured pressure is proportional to the component of momentum along the probe axis over a broad range of flow angles. (This is in contrast to the typical requirement that a pitot probe be insensitive to flow angle.) If, instead, the probe were sensitive to the magnitude of the momentum vector, it would be required also to determine the flow angle in order to resolve the component in the desired direction. If the probe shape can automatically resolve the desired component of momentum, there would be no need to separately determine the flow angle.

\section{Desired Flow Angle Sensitivity ${ }^{2}$}

Consider an axisymmetric pitot probe immersed in a flow of angle, $\alpha$, as illustrated in Fig. 1. The pressuresensing orifice is on the axis of the probe at the probe tip.

\footnotetext{
* The research reported herein was performed by the Arnold Engineering Development Center (AEDC), Air Force Materiel Command. Work and analysis for this research were performed by faculty and students at the United States Air Force Academy and by personnel of Aerospace Testing Alliance, the operations, maintenance, information management, and support contractor for AEDC. Further reproduction is authorized to satisfy needs of the U. S. Government.

${ }^{\dagger}$ Technical Fellow, ATA, AIAA Senior Member

${ }^{\ddagger}$ Engineer/Scientist, ATA

$\S$ Instructor, Department of Aeronautics, HQ USAFA/DFAN, USAF Academy, CO 80840

${ }^{* *}$ Professor, Department. of Aeronautics, HQ USAFA/DFAN, USAF Academy, CO 80840, AIAA Associate Fellow

$\pitchfork$ Air Force Cadet, CS-22, USAF Academy, CO 80840

$\$$ Air Force Cadet, CS-16, USAF Academy, CO 80840, AIAA Student Member
} 


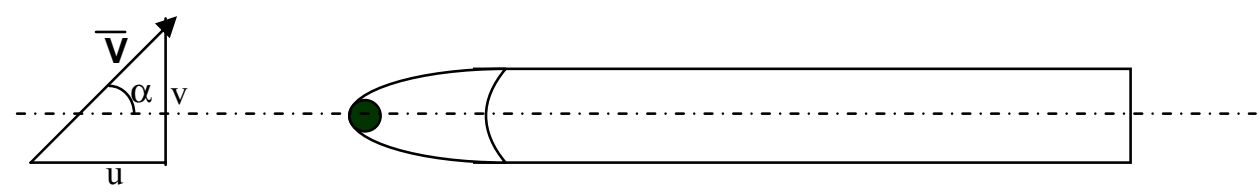

Figure 1. Schematic of Axisymmetric Probe

It is desired that the ratio of the stream thrust $\left(P+\rho u^{2}\right)$ to the pressure sensed by the orifice at the probe tip $\left(P_{m}\right)$ be independent of the flow angle. That is, it is required that

$$
\frac{P+\rho u^{2}}{P_{m}} \neq f(\alpha)
$$

Note that $u$ is the component of velocity along the axis of the probe, whereas $v$ is the component normal to the probe axis. The probe is intended to sense the component of momentum along the probe axis. If the vector, $\boldsymbol{V}$, denotes the flow velocity, then $u$ is given by

$$
u=|\boldsymbol{V}| \cos \alpha
$$

and

$$
u^{2}=|\boldsymbol{V}|^{2} \cos ^{2} \alpha
$$

Thus, the requirement for flow angle independence can be written as

$$
\frac{P+\rho|\boldsymbol{V}|^{2} \cos ^{2} \alpha}{P_{m}} \neq f(\alpha)
$$

with the proviso that $P_{m}$ is also a function of flow angle. It was shown in Ref. 1 that the required flow angle sensitivity is given by

$$
\frac{P_{\alpha}}{P_{0}}=\left[\frac{1+\not M^{2} \cos ^{2} \alpha}{1+\not M^{2}}\right]
$$

where the simplified notation $P_{\alpha}$ is used to indicate the sensed orifice pressure as a function of the flow angle, $\alpha$, and $P_{0}$ is the pitot pressure sensed when the probe is aligned with the flow.

The design problem, therefore, is to determine the probe nose geometry (blunted cone, ogive, ellipse, hemisphere, etc.) that will best approximate the requisite angular dependence as a function of Mach number and flow angle.

It should be noted that Eq. (5) is not simply a $\cos ^{2}$ distribution, as might be expected. However, it obviously approaches this value at high Mach numbers.

\section{CFD Calculations}

In Ref. 2, a CFD code was used to compute the flow about a hemisphere/cylinder and a parabola/cylinder at angles of attack of $0,5,10$, and $15 \mathrm{deg}$ and at Mach numbers of 0.5 and 3.5. A comparison of the nose shapes is shown in Fig. 2. The parabola is given by the equation

$$
y=2 \sqrt{x}-x
$$

whereas the unit hemisphere centered at $(1,0)$ is given by

$$
y=\sqrt{1-(x-1)^{2}}
$$


Note that the parabola is somewhat more blunt than the unit hemisphere. In fact, the radius of curvature at the nose of the parabola is twice that of the hemisphere.

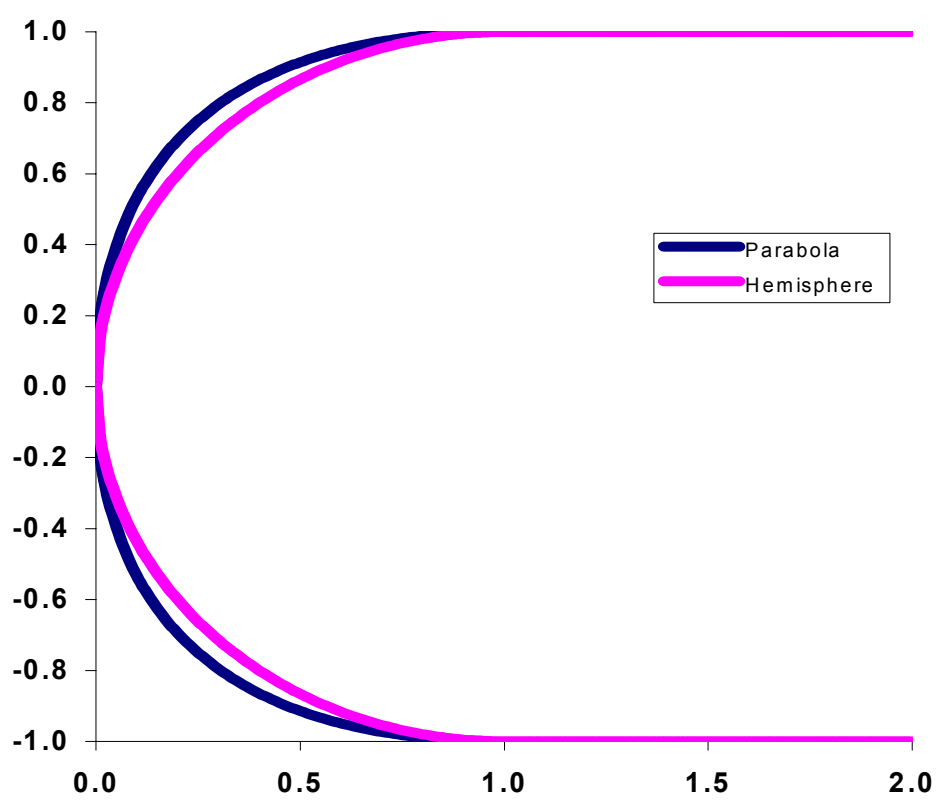

Figure 2. Probe Geometries for CFD Calculations

The Euler CFD solutions previously presented ${ }^{2}$ indicated that, of the two, the parabola/cylinder agreed better with the desired sensitivity. At Mach numbers of 0.5 and 3.5, the parabola/cylinder agreed with the desired distribution within 0.5 percent for flow angles up to $15 \mathrm{deg}$. The maximum disagreement for the hemisphere/cylinder was approximately 0.8 percent.

\section{Description of USAFA Trisonic Wind Tunnel Facility}

Flow angle sensitivity of the parabola/cylinder stream thrust probe was evaluated in the Trisonic Wind Tunnel at the United States Air Force Academy (USAFA). The test section of this blowdown tunnel measures $1 \mathrm{ft}$ square. Specific supersonic test section conditions are set via the use of fixed nozzle blocks; possible tunnel speeds range up to Mach 4.4. Test article pitch angle can be swept across a range from -10 to +10 deg during a test. The Trisonic facility also includes Schlieren video capability.

A schematic of the parabola/cylinder probe tested is shown in Fig. 3. The 3-in.-long probe was mounted on a 7deg-offset "dogleg" sting in order to achieve the desired range of pitch angles from 0 to 15 deg. Figure 4 is a photograph of the probe as mounted in the tunnel.

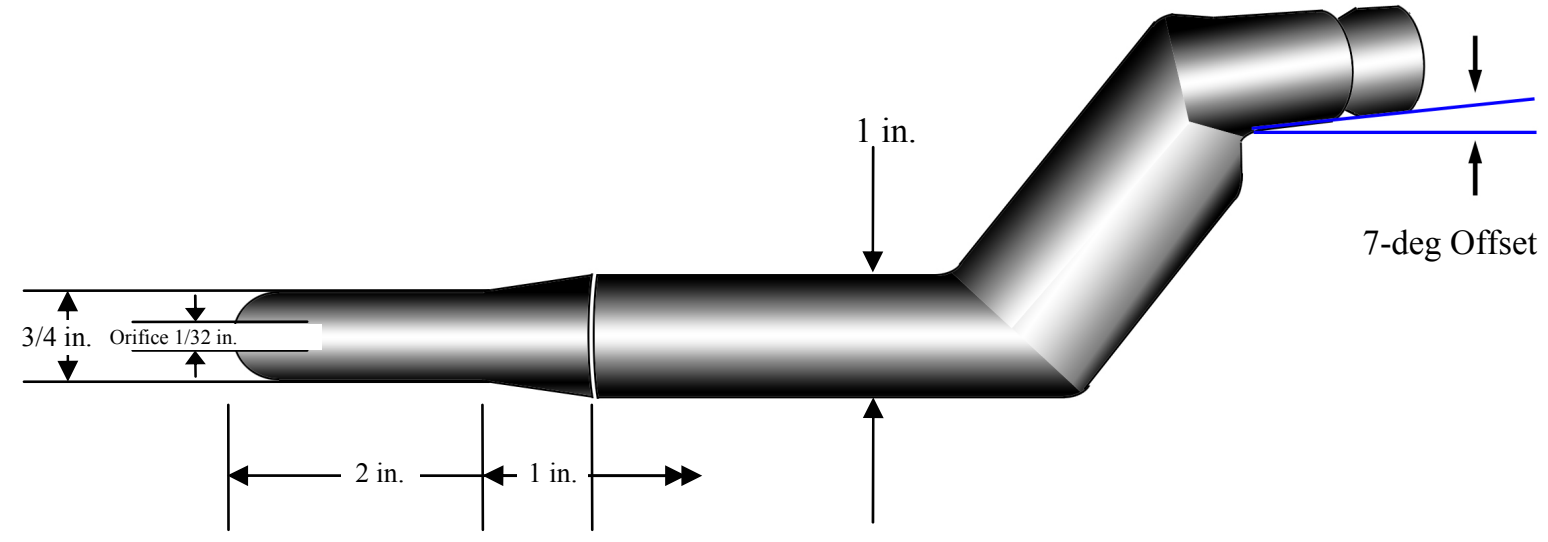

Figure 3. Parabola/Cylinder Stream Thrust Probe 


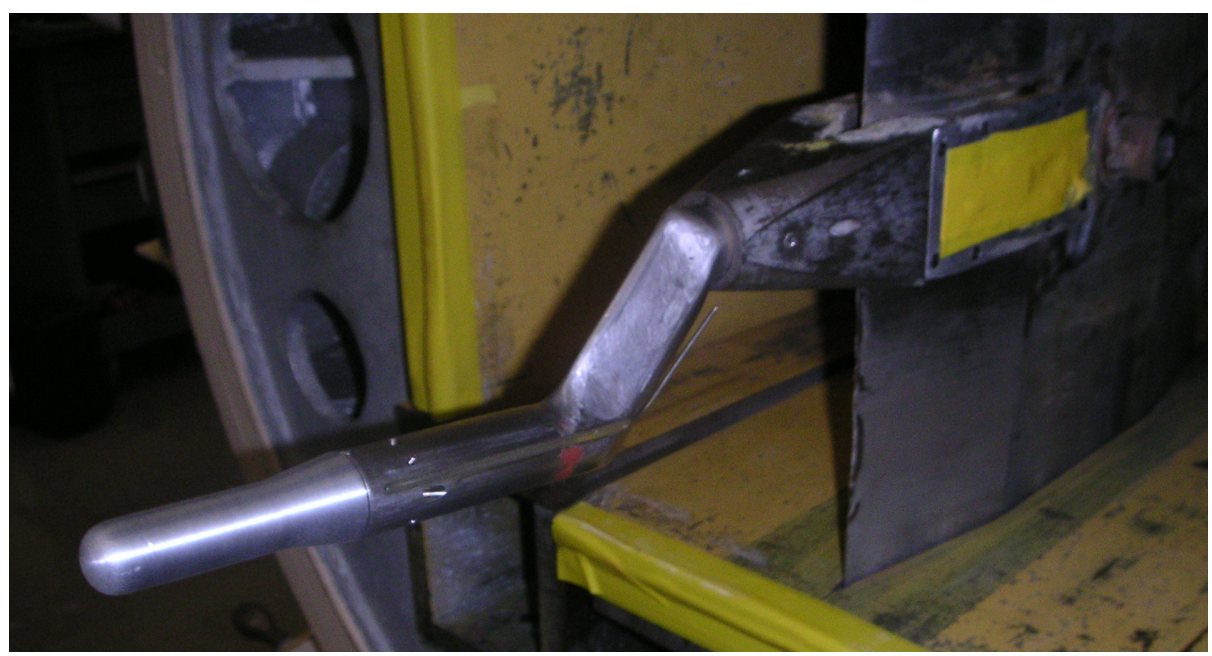

Figure 4. Parabola/Cylinder Probe Mounted in USAFA's Trisonic Wind Tunnel

The probe's sensitivity was measured at tunnel Mach numbers of 1.67, 2.45, and 3.48. At the two lower Mach numbers, the stilling chamber total pressure was set at $50 \mathrm{psia}$; for $3.48 \mathrm{M}$, the setting was 85 psia. Pressures sensed at the orifice were measured by a 50-psid transducer (a 25-psid transducer for the $3.48 \mathrm{M}$ test condition) via $10 \mathrm{ft}$ of plastic tubing. At each probe pitch angle, after a flow stabilization delay of $1.2 \mathrm{~s}$, the pressure was sampled at $100 \mathrm{~Hz}$ for $1 \mathrm{~s}$. Each pressure sample was normalized by the corresponding stilling chamber total pressure, and these 100 normalized pressure samples were averaged. The normalized, averaged probe pressures at each pitch angle were compared to the pressure for the zero-pitch condition. The complete test matrix is shown in Table 1.

Table 1. Flow Angle Sensitivity Test Matrix, Parabola/Cylinder Probe

\begin{tabular}{||c|c|c|c|c||}
\hline \hline Run No. & Mach No. & Date & $\mathrm{P}_{\text {still, }}$ psia & $\mathrm{P}_{\text {atm }}, \mathrm{psia}$ \\
\hline 1 & 1.67 & 10 Mar 04 & 50 & 11.39 \\
\hline 2 & 1.67 & 11 Mar 04 & 50 & 11.42 \\
\hline 3 & 3.48 & 16 Mar 04 & 85 & 11.35 \\
\hline 4 & 3.48 & 16 Mar 04 & 85 & 11.35 \\
\hline 5 & 3.48 & 16 Mar 04 & 85 & 11.33 \\
\hline 6 & 3.48 & 16 Mar 04 & 85 & 11.33 \\
\hline 7 & 2.45 & 18 Mar 04 & 50 & 11.39 \\
\hline 8 & 2.45 & 18 Mar 04 & 50 & 11.40 \\
\hline 9 & 2.45 & 18 Mar 04 & 50 & 11.37 \\
\hline
\end{tabular}

\section{Data Analysis}

The experimental results (and, at Mach 3.48, the CFD predictions) are compared to the desired flow angle sensitivity in Figs. 5 through 7. Uncertainty in the pressure ratio measurements was on the order of \pm 0.5 percent. The test data characterizing the behavior of the parabola/cylinder probe show that the probe overshoots the desired sensitivity - that is, undercompensates for flow angle - at all three supersonic Mach numbers. Third-order polynomial curve fits to the data deviate from the desired sensitivity by as much as 0.94 percent at Mach 1.67, 1.0 percent at Mach 2.45, and 0.98 percnet at Mach 3.48, with the greatest deviations occurring at the highest flow angle. Although, as expected, the probe's behavior follows the correct trend, the deviations from the desired sensitivity exceed the roughly quarter-percent level that is desired so that the influence of flow angle on the overall stream thrust uncertainty is on the same order as the uncertainty in the measured pitot pressure.

It is observed that, at flow angles of $2 \mathrm{deg}$, the measured pressure ratio $P_{d} / P_{0}$ frequently exceeded unity. That is, the pressure sensed by the probe at a 2-deg pitch angle was greater than the pressure sensed at zero pitch. It is expected that, at zero pitch, the pressure sensed by the orifice at the probe tip should be equal to the total pressure of the flow downstream of the shock and, thus, the highest pressure acting anywhere on the probe. That higher pressures are then measured when the probe is pitched is puzzling. Although the pressure ratio increase admittedly is 
small—far less than 1 percent—it occurs frequently enough to deserve investigation. Several possible explanations are proposed.

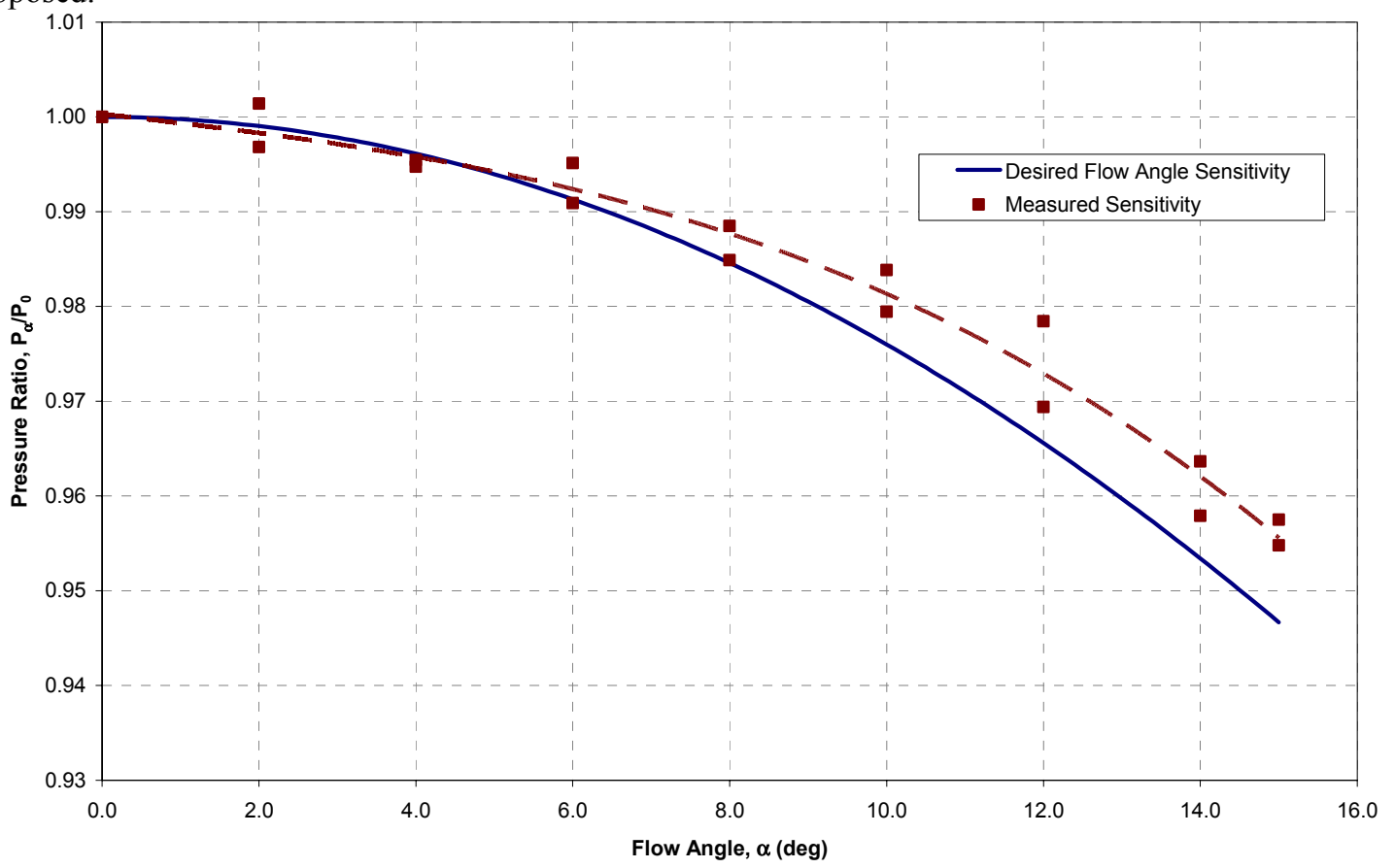

Figure 5. Flow Angle Sensitivity, Parabola/Cylinder Stream Thrust Probe, USAFA Trisonic Wind Tunnel, Mach 1.67

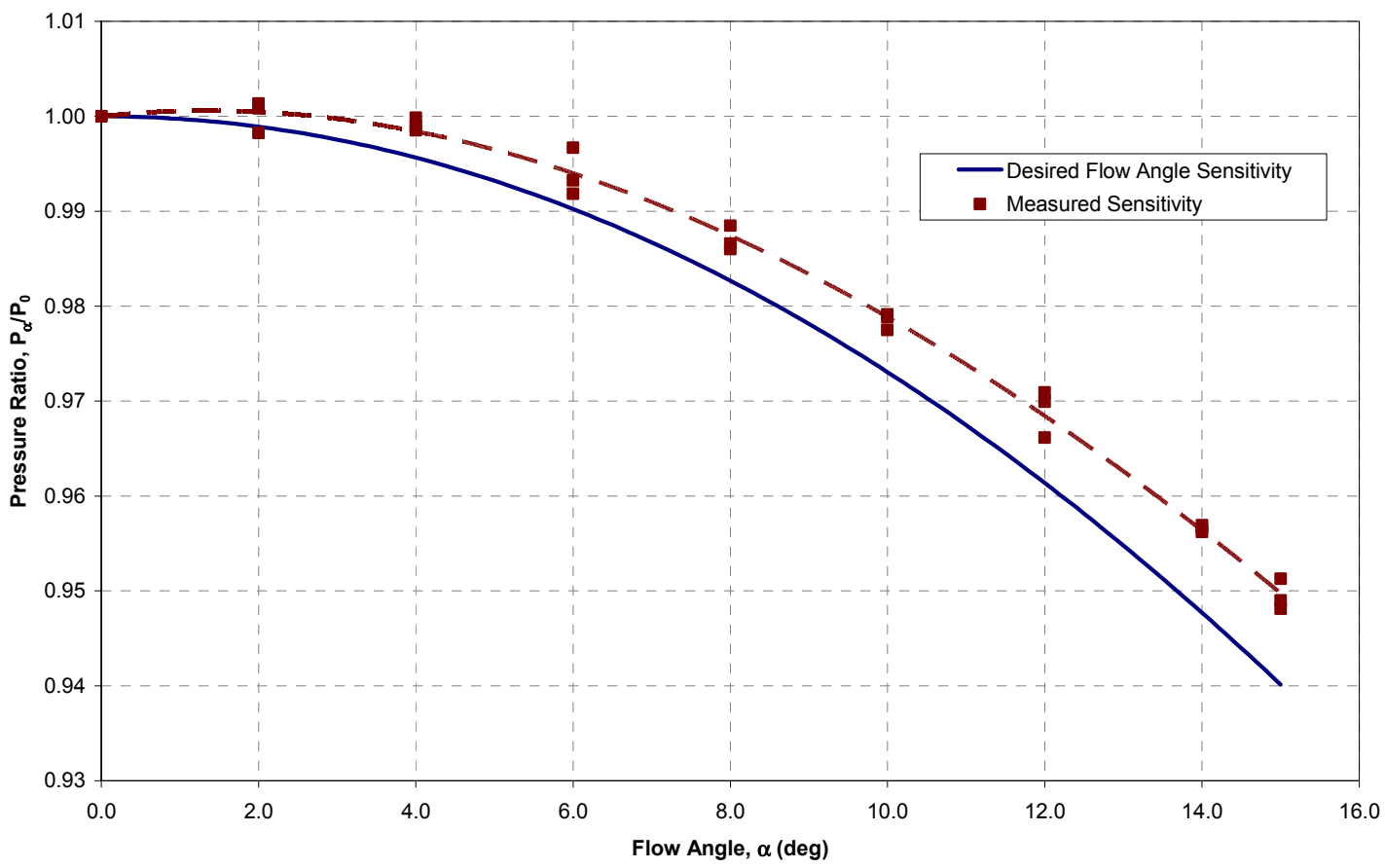

Figure 6. Flow Angle Sensitivity, Parabola/Cylinder Stream Thrust Probe, USAFA Trisonic Wind Tunnel, Mach 2.45 


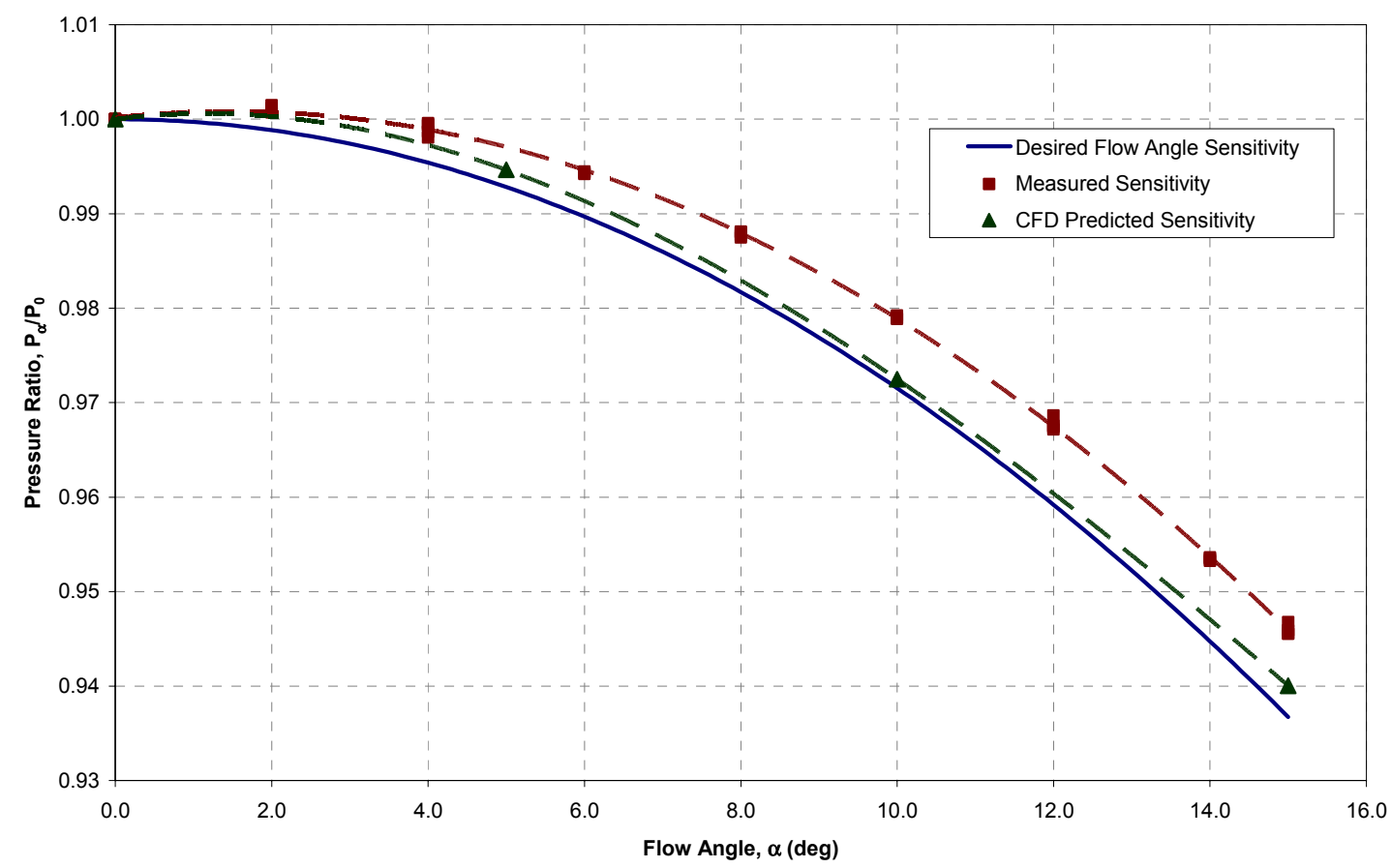

Figure 7. Flow Angle Sensitivity, Parabola/Cylinder Stream Thrust Probe, USAFA Trisonic Wind Tunnel, Mach 3.48

The most obvious potentiality is that some flow angularity (and/or probe axis misalignment) existed in the Trisonic Wind Tunnel test section. However, if such were true, the zero-pitch condition would not correspond to the zero-flow-angle condition, and the wrong reference $P_{0}$ would be used for evaluating probe flow angle sensitivity. In fact, one set of Mach 1.67 data was renormalized using the 2-deg pressure as the reference pressure $P_{0}$. The renormalized data are shown in Fig. 8. (The data were also arbitrarily shifted 1 deg to the left.) That the resulting flow angle sensitivity matches the desired sensitivity so well may indicate the presence of a slight (one- to two-deg) angularity in the flow or probe misalignment; however, justification of the data shift is problematic.

In an attempt to further address this concern, the facility's Schlieren capability was used. A frame of Schlieren video from a zero-pitch test was inverted and then overlaid upon a copy of the same image, allowing comparison of the shock patterns in the top and bottom halves of the flow. No obvious top-to-bottom asymmetries in the shock patterns were apparent, indicating that no large flow angularity was occurring in the test section.

Another possibility is that slight test article imperfections exist, either on the probe's outer surface or at the tip orifice, which might influence surface pressures differently at varying angles of attack. However, subsequent testing with similar probes - provided by a different fabricator-has consistently revealed the same trend of higher pressures at 2-deg pitch angle; conversely, probe axial asymmetries would be expected to cause differing results, depending on the unique locations of test article imperfections.

It should certainly be repeated that instrumentation bias error and precision error resulted in uncertainties in the pressure ratio $P_{d} / P_{0}$ on the order of \pm 0.5 percent. The pressure ratio rise at 2 deg falls well within this experimental uncertainty. For this reason, it is not possible to say for certain that the unexpected pressure ratio rise is even "real"; but if it is real, because the magnitude of the increase is so small, any or all of these or other factors could be just significant enough to induce this behavior. Because of the consistency of the occurrence of this phenomenon, though, further investigation is clearly warranted.

The Mach 3.48 CFD results, shown in Fig. 7, correctly predict that the parabola/cylinder probe's flow angle sensitivity will overshoot the desired sensitivity curve, but the magnitude of the predicted deviation is significantly lower than the measured deviation. 


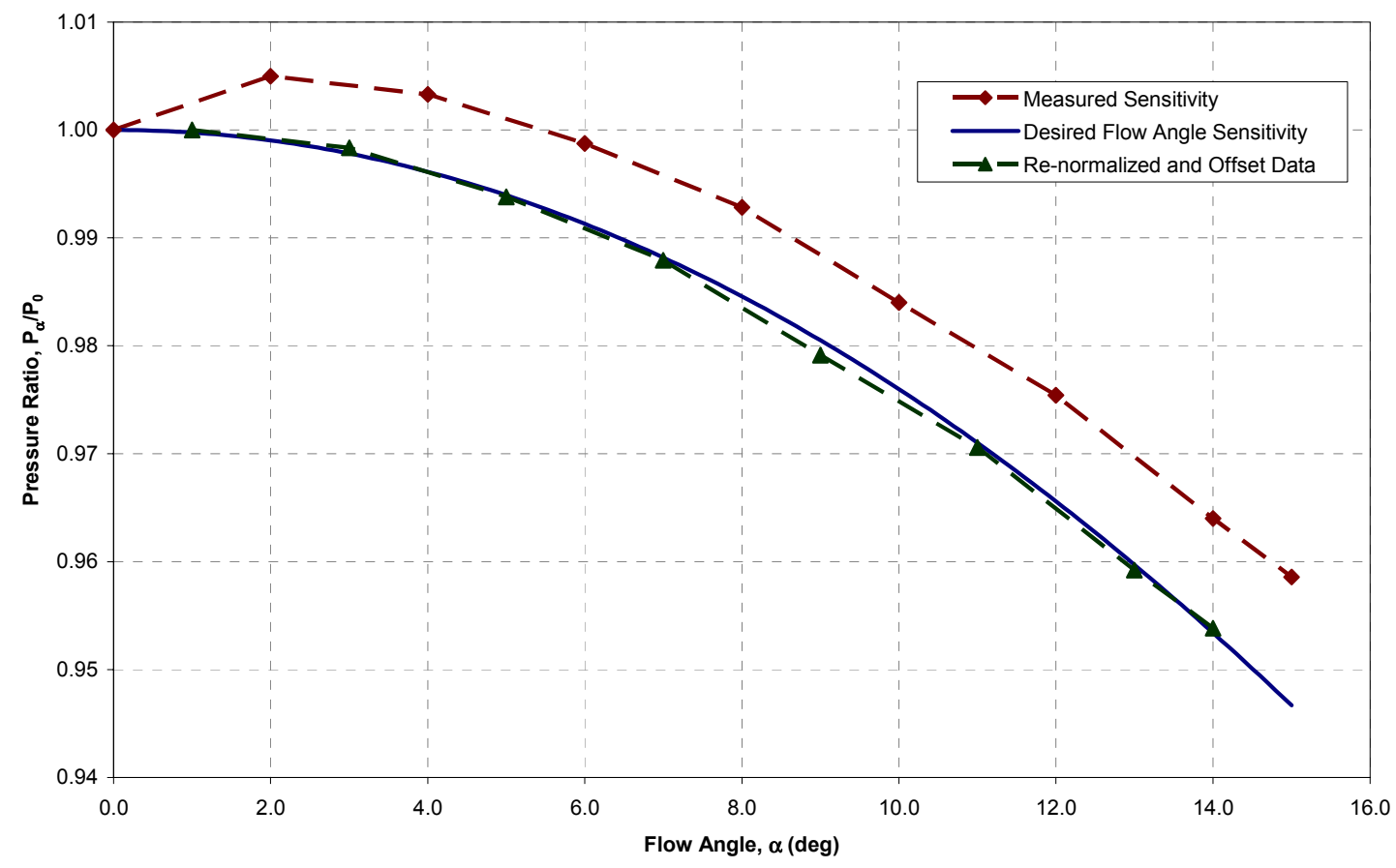

Figure 8. Renormalized Flow Angle Sensitivity, Parabola/Cylinder Stream Thrust Probe, USAFA Trisonic Wind Tunnel, Mach 1.67

As mentioned previously, the CFD simulations reported in Ref. 2 compared the behavior of the parabola/cylinder probe to that of a hemisphere/cylinder probe. The computational results predict that the parabola/cylinder will outperform the hemisphere/cylinder - that is, the parabola/cylinder will better match the desired flow angle sensitivity. The validity of this prediction remains to be demonstrated in a future test series; the relative measured performance of the two probes could then be used to further refine the optimal probe geometry. A computational probe shape optimization study is also under way by researchers at the California Polytechnic State University.

\section{Conclusions}

An experimental evaluation of the flow angle sensitivity of the proposed parabola/cylinder stream thrust probe was conducted in the US Air Force Academy's Trisonic Wind Tunnel across a range of supersonic Mach numbers. The tests indicated that the parabola/cylinder probe exhibits the desired sensitivity reasonably well, undercompensating by no more than 1 percent when flow angle reaches 15 deg. CFD modeling of the parabola/cylinder geometry also predicts undercompensation, though only on the order of a half-percent.

An unexpected pressure ratio rise was frequently observed at $2 \mathrm{deg}$. Though possible explanations, including test section flow angularity, probe misalignment, and test article imperfections, were proposed, none has conclusively been shown to explain the test results. Further investigation, especially into the effect of a possible aggregate probe/ flow misalignment, is warranted.

Wind tunnel testing of the flow angle sensitivity of the hemisphere/cylinder probe shape will be documented in a future report. A comparison of the sensitivities of the parabola/cylinder probe and the hemisphere/cylinder probe may guide future optimization of stream thrust probe geometry, in an attempt to match the desired flow angle sensitivity within 0.25 percent. Computational optimization of probe nose shape is being conducted by Cal Poly researchers in parallel and will influence future experimental work.

\section{Acknowledgments}

The authors wish to recognize the invaluable contributions of the late Larry Lamblin, long-time facility director of the USAFA Trisonic Wind Tunnel. 


\section{References}

${ }^{1}$ Hiers, R. S., and Pruitt, D. W., "Determination of Thrust from Pitot Pressure Measurements," AIAA 20013314, $38^{\text {th }}$ AIAA/ASME/SAE/ASEE Joint Propulsion Conference and Exhibit, 8-11 July 2001.

${ }^{2}$ Hiers, R. S., and Sirbaugh, J. R., "The Aerodynamics of Stream Thrust Probes," AIAA 2003-1092, 41st Aerospace Sciences Meeting and Exhibit, 6-9 January 2003. 